Revue d'histoire de l'enfance « irrégulière »

Le Temps de l'histoire

$17 \mid 2015$

Naissance et mutation de la justice des mineurs

\title{
Du côté du CNAHES : les archives de l'éducation spécialisée ont désormais leurs quartiers à Pierrefitte!
}

Sylvain Cid

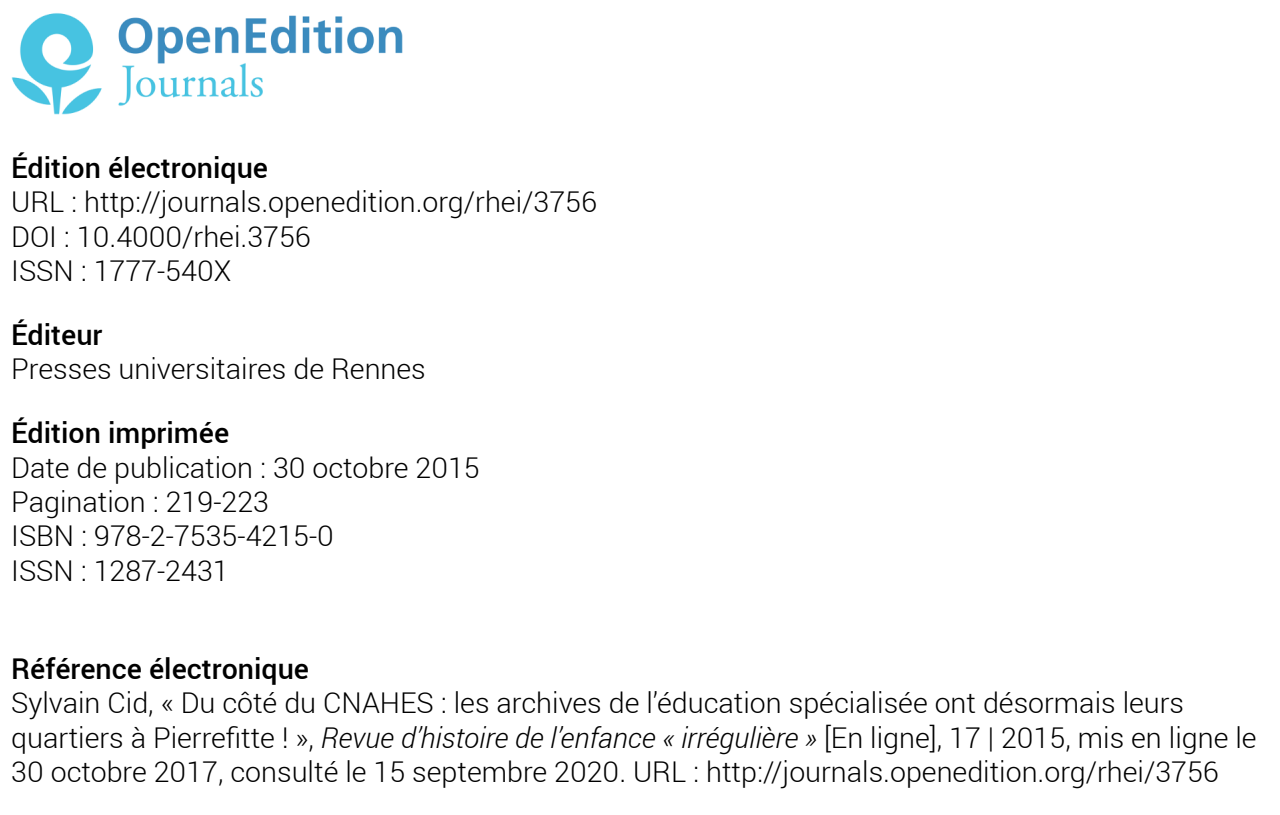

(C) PUR 


\section{Du côté du CNAHES: les archives de l'éducation spécialisée ont désormais leurs quartiers à Pierrefitte!}

Mots-clefs : archives, éducation spécialisée, Pierrefitte

Keywords : archives, special education, Pierrefitte

$\mathrm{D}$ u 23 au 25 septembre 2014, ce sont 46 fonds d'archives collectés par le CNAHES $^{1}$ depuis 20 ans, soit 438,65 mètres linéaires de documents, qui ont été déménagés des Archives nationales du monde du travail (ANMT) à Roubaix au site des Archives nationales de Pierrefitte-sur-Seine. Cette opération menée en accord avec les Archives de France était motivée par plusieurs bonnes raisons, à la fois liées au sens que nous souhaitions donner à ce patrimoine et aux opportunités d'animation, de formation et de recherche qu'elle pouvait susciter. Elle a répondu tout d'abord au ferme désir que nous avions de rapprocher ces fonds privés des archives publiques des administrations centrales qui avaient exercé leur contrôle sur ce large secteur associatif « habilité ». Nous y avons vu l'opportunité de mettre l'accent sur une complémentarité historique " public-privé » constitutive de ce champ. Nous avons voulu défendre que, loin de se réduire à l'aventure d'une initiative privée déconnectée des grands enjeux nationaux, l'histoire " privée » des personnes comme des associations auxquelles nous nous intéressons tient au contraire une large place dans l'histoire des politiques sociales, éducatives et judiciaires. Depuis le $\mathrm{XIX}^{\mathrm{e}}$ siècle rappelonsle, les œuvres privées représentent entre les deux tiers et les trois quarts de l'équipement. Par ailleurs, le rapprochement avec le Pôle des archives de la jeunesse et de l'éducation populaire (PAJEP), dont une partie des archives est également accueillie à Pierrefitte, devait permettre aussi de soutenir une autre proximité parfois oubliée: celle des politiques menées respectivement jusqu'à présent en direction de la " jeunesse qui va bien " et de la « jeunesse qui va mal ». Un tel transfert serait également avantageux pour les associations natio-

\section{Sylvain CID \\ Archiviste au \\ Conservatoire national \\ des archives et de \\ I'histoire de l'éduca- \\ tion spécialisée et \\ de l'action sociale \\ (CNAHES).}

\section{Le CNAHES est le}

Conservatoire national des archives et de l'histoire de l'éducation spécialisée et de l'action sociale (siège social: 63 rue Croulebarbe -75013 Paris, tél. : 07864856 24, courriel: info@cnahes.org, site Internet: [www.cnahes. org]). 
nales déposantes, mobilisées au niveau de la collecte et de la valorisation de leurs archives, dont le siège est le plus souvent francilien. Il offrait enfin l'opportunité de dynamiser la formation et la recherche autour de ce patrimoine grâce à la demande potentiellement plus forte des formations de travailleurs sociaux et des universités.

Les déposants ou donateurs des fonds collectés peuvent être des personnes physiques ou morales. En premier lieu viennent les " acteurs" eux-mêmes, d'horizons et de profils différents. On trouve parmi eux des éducateurs spécialisés ou assimilés (Jacques Guyomarc’h, Serge Ginger et Jacques Leblanc, Jean Ughetto, etc.), des instituteurs (Simonne et Jacques Lacapère), des assistants sociaux (Roland Assathiany, Vivette Samuel), des psychiatres (Louis Le Guillant, Maurice Parienté, Victor Girard, Georges Heuyer), des magistrats (Pierre Mercier, Marcel Guilloteau), des directeurs de centres de l'Éducation surveillée (René Courtois, Dominique Riehl), une inspectrice de l'Éducation surveillée (Marie Mauroux-Fonlupt) et un inspecteur de l'Éducation nationale (André Catteaux). Du côté des éducateurs spécialisés ou des juges, l'ancrage territorial est parfois important: leurs fonds respectifs apportent un éclairage sur la configuration particulière d'une région (Jacques Guyomarc'h pour la Bretagne, Paul Bertrand pour le Pays basque...), ou d'un ancien protectorat ou colonie (André Heinrich pour le Maroc, Jacques Gauneau pour l'Algérie). En second lieu, les associations professionnelles ont aussi produit une grande quantité d'archives qui peuvent témoigner d'un rôle joué à la fois de construction et de contrôle à l'intérieur, de représentation et de défense à l'extérieur. Se comptent parmi elles l'Association nationale des éducateurs de jeunes inadaptés (ANEJI), association majoritaire, à côté d'une très faible syndicalisation, des éducateurs spécialisés de sa création en 1947 jusqu’à son extinction en 1993; la Confédération française des professions sociales (CFPS), issue de l'ancienne Association des travailleuses sociales (ATS) et porteuse avec un succès inégal du projet de rassembler sous sa bannière toutes les professions sociales; l'Association française des magistrats de la jeunesse et de la famille (AFMJF), créée en 1948. L'ANEJI et l'AFMJF sont chacune doublées d'une association internationale dont le CNAHES a aussi collecté quelques archives. En troisième lieu s'ajoute potentiellement le grand nombre des associations gestionnaires, des établissements et services privés dont l'activité s'inscrit dans l'éducation spécialisée, mais dont nous ne prétendrons jamais réaliser la collecte exhaustive. Il s'agit pour partie des défunts centres 
d'observation (CO), des centres de rééducation, des instituts médico-éducatifs (IME) et centres d'aide par le travail (CAT), des maison d'enfants à caractère social (MECS)... Généralement, les archives de ces établissements ont été traitées en même temps que les archives des associations qui les géraient. Parmi celles-ci, les Associations régionales de sauvegarde de l'enfance et de l'adolescence (ARSEA) tiennent une place importante à côté néanmoins de bien d'autres. Ces associations gestionnaires sont elles-mêmes souvent fédérées au niveau national par des unions d'associations influentes et également très productrices d'archives. Le CNAHES est dépositaire des fonds de l'Union nationale des associations régionales de sauvegarde de l'enfance et de l'adolescence (UNAR), de l'Union nationale interfédérale des organismes privés sanitaires et sociaux (UNIOPSS), de l'Association nationale d'entraide féminine (ANEF) et de la Fédération générale des pupilles de l'enseignement public (FGPEP). Le tableau ne serait pas complet sans les centres de formation aux professions éducatives et sociales (éducateurs spécialisés, moniteurs-éducateurs, assistants sociaux...) aussi majoritairement privés et dont quelques fonds sont classés (École normale sociale à Paris, Recherches et Promotion à Lyon...). Ces centres de formation se sont eux aussi regroupés au sein de comités d'entente, puis d'unions d'associatives successives ${ }^{2}$ dont une partie des archives est aussi traitée.

Parmi tous ces fonds, une partie seulement est véritablement consultable à Pierrefitte. Cela tient tout d'abord très logiquement à ce que le site de Pierrefitte est un centre d'archives nationales. Au terme d'une convention signée en 2002 entre le CNAHES et les ministères chargés respectivement de la culture, de la justice et des affaires sociales, il a été prévu que les fonds de portée locale ou régionale seraient déposés dans les services d'archives territoriales compétents, en premier lieu les archives départementales. Pour cette raison, on ne trouvera à Pierrefitte que peu d'archives d'établissements et aucun dossier de jeune. Celles-ci ont le plus souvent été repérées, traitées et inventoriées grâce à l'intervention spécifique des délégations régionales du CNAHES, actuellement au nombre de 11. Par ailleurs, pour des raisons différentes, tous les fonds de portée nationale traités et/ou pris en charge par le CNAHES n'ont pas (encore) été acheminés à Pierrefitte. Cette situation procède parfois du choix même d'associations qui préfèrent assurer elles-mêmes la conservation de leurs archives. Rappelons ici au passage que certaines institutions nationales privées n'ont pas attendu le CNAHES pour organiser la conservation et l'accès de leurs archives:
2 Le CNAHES a recueilli les archives de I'UNITES (Union nationale des instituts de formation en travail éducatif et social), ancienne union majoritaire des centres de formation de travailleurs sociaux, aujourd'hui réunis en totalité au sein de l'UNAFORIS (Union nationale des associations de formation et de recherche en intervention sociale). 
3 Archives des Orphelins apprentis d'Auteuil: 40 rue Jean de la Fontaine. Archiviste: Marie-Noëlle Dumont. Tél. : 01441474 77 ; courriel: marie-noelle. dumont@apprentis-auteuil.

4 Archives de la MaisonMère du Bon Pasteur: Maison-Mère du Bon Pasteur,

Archives, 18 rue Marie-

Euphrasie Pelletier, 49100 Angers. Archiviste depuis 2010: Sarah Elbisser. Tél. :

0241721240 (accueil de la Maison-Mère); courriel: archivistemm@gmail. com. Archives provinciales (province BFMN, Belgique France Magyarorszag Nederland): 3 impasse de Tournemine, 49100 Angers. Archiviste depuis 2010: Sibylle Gardelle. Tél. :

0241721240 ; courriel: archives@bon-pasteur-bfm. org. Site internet: [www.bonpasteur-bfm.org].
5 Archives générales et provinciales de Notre-Dame de Charité du Refuge: Union Notre-Dame de Charité - 2 Rue de la Charité 14123 Cormelles-le-Royal. Archiviste depuis 2005: $\mathrm{M}^{\text {me }}$ Delphine Le Crom. Tél. 0231355040 ; courriel: archivesndc@yahoo.fr.

6 Archives générales, provinciales, de la Maison Mère et archives économiques: Maison Mère des Filles de la Sagesse, 3 rue Jean-Paul II, BP 79, 85292 St Laurent sur Sèvre cedex. Archiviste depuis 2004: Sœur AnneMarie Le Port. Tél. : 025164 3800 ; courriel: fdls.archives. mm@orange.fr.

7 [www.siv.archives-nationales.culture.gouv.fr]. ce sont par exemple les Apprentis d'Auteuil ${ }^{3}$, mais aussi et surtout des congrégations comme le Bon Pasteur d'Angers ${ }^{4}$, le Refuge de Caen ${ }^{5}$ et les Filles de la Sagesse ${ }^{6}$. Dans les autres cas, l'entrée d'un fonds au centre de Pierrefitte nécessite non seulement la réalisation des tri, classement et inventaire indispensables à sa consultation, mais aussi son acceptation finale par les Archives nationales. Deux instances, un comité de suivi et une commission d'entrée des fonds, ont pour rôle d'instruire et de statuer sur ces entrées " par voix extraordinaire " proposées par le CNAHES. Ces dernières années, le projet de transfert des archives de Roubaix à Pierrefitte a retardé l'entrée régulière des autres fonds d'archives de portée nationale. Aujourd'hui, environ 350 mètres linéaires supplémentaires attendent encore leur entrée à Pierrefitte.

Le déménagement à Pierrefitte marque un nouveau départ riche en collaborations nouvelles et prometteuses. En lien avec les Archives nationales, une première étape de travail à venir va consister à actualiser et adapter nos inventaires d'archives à de nouvelles règles de cotation et d'écriture (encodage en EAD-XML avant intégration dans la salle d'inventaires virtuelle des Archives nationales $\left.{ }^{7}\right)$. Elle sera suivie par l'intégration des fonds de portée nationale restés en attente dans différents lieux provisoires de conservation. À moyen terme, nous souhaitons redéployer notre collecte en direction de terrains encore peu explorés comme celui du handicap ou de la prévention spécialisée. La valorisation des archives orales constituées jusqu'à présent par des chercheurs ou d'autres membres actifs du CNAHES devrait quant à elle trouver un aboutissement grâce à un partenariat que nous 
initions avec le département audiovisuel de la Bibliothèque nationale de France. À l'occasion de la célébration du $20^{\mathrm{e}}$ anniversaire du CNAHES à Pierrefitte, un premier recensement des archives privées de l'éducation spécialisée et de l'action sociale a été présenté. Il représente la première version d'un guide des sources dont nous souhaitons qu'il encouragera encore davantage les étudiants, chercheurs, élèves de centres de formation... à venir s'emparer de ce champ de recherche encore neuf. 